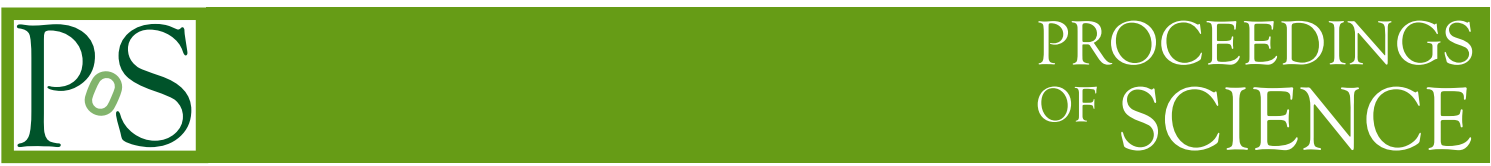

\title{
WIMP dark matter and baryogenesis
}

\author{
Nicolás Bernal* \\ Bethe Center for Theoretical Physics and Physikalisches Institut, \\ Universität Bonn, Nußallee 12, D-53115 Bonn, Germany \\ E-mail: nicolaseth.physik.uni-bonn.de
}

\section{François-Xavier Josse-Michaux}

Centro de Física Teórica de Partículas CFTP, Instituto Superior Técnico,

Technical University of Lisbon, 1049-001 Lisboa, Portugal

E-mail: fxjossemichaux@gmail.com

\section{Lorenzo Ubaldi}

Bethe Center for Theoretical Physics and Physikalisches Institut,

Universität Bonn, Nußallee 12, D-53115 Bonn, Germany

E-mail: ubaldi@th.physik.uni-bonn.de

\begin{abstract}
A possible connection between the abundances of baryonic and dark matter (DM) has been explored so far mostly in the context of the so-called asymmetric DM. Recently, a very different mechanism, dubbed "WIMPy baryogenesis", has been proposed to relate the baryon asymmetry to DM annihilation. The DM candidate is a weakly interacting massive particle (WIMP), and the usual WIMP scenario is slightly extended to accommodate baryogenesis, which is accomplished around the time of DM freeze-out. We construct an effective field theory that encompasses a quite general class of models which implement the WIMPy baryogenesis. Under some reasonable, simplifying assumptions, we show that a good portion of the parameter space is allowed for these models, after experimental constraints are taken into account. Bounds from the LHC require that the WIMP be heavier than $500 \mathrm{GeV}$.
\end{abstract}

Proceedings of the Corfu Summer Institute 2012 "School and Workshops on Elementary Particle Physics and Gravity"

September 8-27, 2012

Corfu, Greece

\footnotetext{
*Speaker.
} 


\section{Introduction}

The presence of non-luminous and non-baryonic matter, the so-called Dark Matter (DM) [1, 2, $3,4,5,6,7]$, and the existence of the baryon asymmetry of the Universe (BAU) [8, 9] are two well established facts. Cosmic microwave background (CMB) anisotropy observations by the Planck satellite yield an accurate determination of the total amount of baryonic matter [9],

$$
\Omega_{\mathrm{b}} h^{2}=0.02205 \pm 0.00028
$$

and of non-baryonic matter

$$
\Omega_{\mathrm{DM}} h^{2}=0.1199 \pm 0.0027 .
$$

The fact that the two abundances are comparable $\Omega_{\mathrm{DM}} / \Omega_{\mathrm{b}} \sim 5$ can be fortuitous, or may be the sign that they have a common origin. This intriguing possibility has been vastly explored in the literature, mostly by invoking asymmetric DM scenarios [10, 11, 12, 13]. The common feature of all such models is that the DM abundance is determined by a matter-antimatter asymmetry in the dark sector, which in turn is connected to the baryon asymmetry in the visible sector. Let us emphasize that this kind of models aim to explain the factor of $a$ few between this two quantities.

Different approaches have been proposed where the DM candidate is given by just one weakly interacting massive particle (WIMP), and where always the dark sector is minimally extended in order to accomplish the BAU [14, 15, 16, 17, 18, 19]. In particular, in reference [17] a new mechanism called "WIMPy baryogenesis" has been presented: On top of the DM WIMP, one heavy particle $(\sim \mathrm{TeV})$ charged under the Standard Model $(\mathrm{SM})$ quantum numbers, $\psi$, and one very light and uncharged particle $n$ are introduced. DM annihilates into $\psi$ and a quark, and $\psi$ subsequently decays into the light, sterile state $n$, storing the negative asymmetry in baryon number in a sequestered sector. The DM relic density is that of a thermal WIMP, and by the time the DM annihilations freeze out one has generated a baryon asymmetry, when the annihilation is into $\psi$ plus quark. Baryogenesis can occur after the electroweak phase transition and masses down to a few hundreds $\mathrm{GeV}$ are allowed.

The aim of the current paper is to review a general class of models that implements the WIMPy baryogenesis mechanism. In the spirit of keeping the models as simple as possible we identify the minimal particle content that does the job, and in the framework of an effective field theory (EFT) we find the complete set of four-fermion interaction terms (Section 2). In section 3 we describe the evolution of the DM and the BAU, via the study of the Boltzmann equations. Sections 4 to 6 are devoted to the discussion of the experimental constraints from the LHC, cosmology and DM direct detection. We summarize our conclusions in section 7. In reference [18] the reader can find more details about this study.

\section{An effective-field-theory approach}

\subsection{Field content of the model and Lagrangian}

We add to the SM the minimal particle content which is needed in order to have a successful WIMPy baryogenesis. All the new particles are fermions. We consider vector-like gauge singlet 


\begin{tabular}{|c|c|c|c|c|c|}
\cline { 2 - 6 } \multicolumn{1}{c|}{} & $S U(3)_{c}$ & $S U(2)_{L}$ & $Q_{U(1)_{y}}$ & $Q_{U(1)_{B}}$ & $\mathbb{Z}_{4}$ \\
\hline$X$ & 1 & 1 & 0 & 0 & $+i$ \\
$\bar{X}$ & 1 & 1 & 0 & 0 & $-i$ \\
$\psi$ & 3 & 1 & $+2 / 3$ & $+1 / 3$ & +1 \\
$\bar{\psi}$ & $\overline{3}$ & 1 & $-2 / 3$ & $-1 / 3$ & +1 \\
$n$ & 1 & 1 & 0 & 0 or +1 & +1 \\
$\bar{u}$ & $\overline{3}$ & 1 & $-2 / 3$ & $-1 / 3$ & -1 \\
$\bar{d}$ & $\overline{3}$ & 1 & $+1 / 3$ & $-1 / 3$ & -1 \\
\hline
\end{tabular}

Table 1: Particle content of the model. $\bar{u}$ and $\bar{d}$ are the right-handed up and down quarks of the SM. The rest of the $S M$ quarks also have charge -1 , while all the leptons are neutral under the $\mathbb{Z}_{4}$ symmetry.

DM $X$ and $\bar{X}$, vector-like exotic quark color triplets $\psi$ and $\bar{\psi}$, and a massless singlet $n$, into which the exotic quark decays.

A discrete symmetry is needed in our model, in order to stabilize the DM and to avoid dangerous decays of the exotic quark that could spoil the baryon asymmetry. Note that all the SM quarks carry charge -1 under the $\mathbb{Z}_{4}$, while all the leptons and the Higgs boson are neutral, so that the familiar renormalizable SM Lagrangian is unchanged.

Given the particle content of the model (table 1), we want to write down all the possible dimension six operators. Our Lagrangian has the form

$$
\begin{aligned}
\mathscr{L} \supset & \frac{1}{\Lambda^{2}} \sum_{i} \lambda_{i}^{2} \mathscr{O}_{i}, \\
= & \frac{1}{\Lambda^{2}}\left[\lambda_{1}^{2}(X X)(\psi \bar{u})+\lambda_{2}^{2}(\bar{X} \bar{X})(\psi \bar{u})+\lambda_{3}^{2}\left(X^{\dagger} X^{\dagger}\right)(\psi \bar{u})+\lambda_{4}^{2}\left(\bar{X}^{\dagger} \bar{X}^{\dagger}\right)(\psi \bar{u})\right. \\
& +\lambda_{5}^{2}\left(\bar{X}^{\dagger} \bar{\psi}^{\dagger}\right)(X \bar{u})+\lambda_{6}^{2}\left(X^{\dagger} \bar{\psi}^{\dagger}\right)(\bar{X} \bar{u})+\lambda_{7}^{2}(X \bar{u})\left(X^{\dagger} \bar{u}^{\dagger}\right)+\lambda_{8}^{2}(\bar{X} \bar{u})\left(\bar{X}^{\dagger} \bar{u}^{\dagger}\right) \\
& +\lambda_{9}^{2}(\psi \psi)(\bar{u} \bar{u})+\lambda_{10}^{2}(\psi \bar{u})\left(\psi^{\dagger} \bar{u}^{\dagger}\right)+\lambda_{11}^{2}\left(\bar{\psi}^{\dagger} \bar{\psi}^{\dagger}\right)(\bar{u} \bar{u})+\lambda_{12}^{2}\left(\bar{\psi}^{\dagger} \bar{u}^{\dagger}\right)(\bar{\psi} \bar{u}) \\
& +\lambda_{13}^{2}(X \bar{X})(\psi \bar{\psi})+\lambda_{14}^{2}\left(X^{\dagger} \bar{X}^{\dagger}\right)(\psi \bar{\psi})+\lambda_{15}^{2}(X \psi)(\bar{X} \bar{\psi})+\lambda_{16}^{2}\left(X^{\dagger} \bar{\psi}^{\dagger}\right)(X \bar{\psi}) \\
& \left.+\lambda_{17}^{2}\left(\bar{X}^{\dagger} \bar{\psi}^{\dagger}\right)(\bar{X} \bar{\psi})+\lambda_{18}^{2}\left(X^{\dagger} \psi^{\dagger}\right)(X \psi)+\lambda_{19}^{2}\left(\bar{X}^{\dagger} \psi^{\dagger}\right)(\bar{X} \psi)+\lambda_{20}^{2}(\bar{\psi} \bar{d})(\bar{d} n)\right]+ \text { h.c. },(2.1)
\end{aligned}
$$

where the two-component spinor formalism [20] has been used.

In this paper we set the scale $\Lambda$ to $10 \mathrm{TeV}$. The reader should keep in mind that varying the couplings $\lambda_{i}$ 's is equivalent to varying the scale $\Lambda$, the only measurable quantity in an EFT being the combination $\lambda_{i} / \Lambda$.

To keep the number of parameters in the numerical analysis manageable, we set some equalities among the relevant couplings and we relabel them for the ease of the discussion:

- $\lambda_{s 1} \equiv \lambda_{1}=\lambda_{3}, \quad \lambda_{s 2} \equiv \lambda_{2}=\lambda_{4}$ : couplings for DM (or anti-DM) annihilation into $\psi \bar{u}$ and $\psi^{\dagger} \bar{u}^{\dagger}$ in the $s$-channel;

- $\lambda_{t} \equiv \lambda_{5}=\lambda_{6}$ : couplings for DM (or anti-DM) annihilation into $\psi \bar{u}$ and $\psi^{\dagger} \bar{u}^{\dagger}$ in the $t$-channel;

- $\lambda_{\mathrm{WO}} \equiv \lambda_{9}=\lambda_{11}$ : couplings responsible for the "pure" washout processes;

- $\lambda_{n} \equiv \lambda_{20}$ : coupling implying the decay $\psi \rightarrow \bar{d} \bar{d} n$; 
- $\lambda_{\psi \psi} \equiv \lambda_{13}=\lambda_{14}=\lambda_{15}=\lambda_{16}=\lambda_{17}=\lambda_{18}=\lambda_{19}$ : couplings for the annihilation $X \bar{X} \rightarrow \psi \bar{\psi}$;

- $\lambda_{7}$ and $\lambda_{8}$ : couplings responsible for the DM annihilation into two quarks and to DM direct detection.

The couplings $\lambda_{s 1}, \lambda_{s 2}$ and $\lambda_{t}$ are also involved in the "mixed" washout processes, that are related to the annihilations by crossing symmetry; whereas the "pure" washout terms (proportional to $\lambda_{\text {wo }}$ ) involve only $\psi$ and $\bar{u}$.

In our analysis we will consider two limiting cases:

$$
\begin{array}{ll}
\lambda_{s}^{2} \equiv \lambda_{s 1}^{2}=+\lambda_{s 2}^{2} & \text { scalar } s \text {-channel } \\
\lambda_{p}^{2} \equiv \lambda_{s 1}^{2}=-\lambda_{s 2}^{2} & \text { pseudoscalar } s \text {-channel }
\end{array}
$$

Only the couplings appearing in the previous relabeling list are relevant to the following phenomenological study. Let us note that the annihilation channel $X \bar{X} \rightarrow \psi \bar{\psi}$ could be a potentially problematic source of washout of the BAU. In order to avoid this problem, in the numerical analysis that follows we scan for $m_{\psi}$ in the range $[0.8,2] \times m_{\chi}$. Hence the coupling $\lambda_{\psi \psi}$ is completely irrelevant in most of this range, namely between $m_{\chi}$ and $2 m_{\chi}$, because this channel is not kinetically open.

\section{Boltzmann equations}

The evolution of the DM, $\psi$ and baryon asymmetry number densities in the expanding Universe is governed by a set of Boltzmann equations. Introducing the rescaled inverse temperature $z \equiv m_{\chi} / T$ and the comoving number densities $Y_{\xi} \equiv n_{\xi}(z) / s(z), s(z)$ being the entropy density, we can write:

$$
\begin{aligned}
z s(z) H(z) \frac{d Y_{\mathrm{DM}}}{d z}= & -2\left(\gamma_{\mathrm{ann}}^{\mathrm{CPV}}(z)+\gamma_{\mathrm{ann}}^{\mathrm{CPC}}(z)\right)\left(\left(\frac{Y_{\mathrm{DM}}}{Y_{\mathrm{DM}}^{\mathrm{eq}}}\right)^{2}-1\right) \\
z s(z) H(z) \frac{d Y_{\Delta u}}{d z}= & \varepsilon(z) \gamma_{\mathrm{ann}}^{\mathrm{CPV}}(z)\left(\left(\frac{Y_{\mathrm{DM}}}{Y_{\mathrm{DM}}^{\mathrm{eq}}}\right)^{2}-1\right) \\
& -\left(\frac{Y_{\Delta u}}{Y_{u}^{\mathrm{eq}}}-\frac{Y_{\Delta \psi}}{Y_{\psi}^{\mathrm{eq}}}\right)\left(\frac{Y_{\mathrm{DM}}}{Y_{\mathrm{DM}}^{\mathrm{eq}}} \gamma_{\mathrm{WO}}^{m}(z)+2 \gamma_{\mathrm{WO}}^{p}(z)\right) \\
z s(z) H(z) \frac{d Y_{\Delta \psi}}{d z}= & -\varepsilon(z) \gamma_{\mathrm{ann}}^{\mathrm{CPV}}(z)\left(\left(\frac{Y_{\mathrm{DM}}}{Y_{\mathrm{DM}}^{\mathrm{eq}}}\right)^{2}-1\right)+\left(\frac{Y_{\Delta u}}{Y_{u}^{\mathrm{eq}}}-\frac{Y_{\Delta \psi}}{Y_{\psi}^{\mathrm{eq}}}\right)\left(\frac{Y_{\mathrm{DM}}}{Y_{\mathrm{DM}}^{\mathrm{eq}}} \gamma_{\mathrm{WO}}^{m}(z)+2 \gamma_{\mathrm{WO}}^{p}(z)\right) \\
& -\gamma_{D}\left(\frac{Y_{\Delta \psi}}{Y_{\psi}^{\mathrm{eq}}}+2 \frac{Y_{\Delta d}}{Y_{d}^{\mathrm{eq}}}\right) \\
z s(z) H(z) \frac{d Y_{\Delta d}}{d z}= & -2 \gamma_{D}\left(\frac{Y_{\Delta \psi}}{Y_{\psi}^{\mathrm{eq}}}+2 \frac{Y_{\Delta d}}{Y_{d}^{\mathrm{eq}}}\right)
\end{aligned}
$$

where $H(z)$ is the Hubble expansion rate and the $\gamma$ 's correspond to the thermally averaged interaction rates.

The Boltzmann equations are quadratic in the DM density $Y_{\mathrm{DM}} \equiv Y_{X}+Y_{\bar{X}}$, but we only expand them to first order in the asymmetries $\varepsilon$ and $Y_{\Delta \alpha} \equiv Y_{\alpha}-Y_{\bar{\alpha}}$, with $\alpha=u, d, \psi$, as these are expected 
to be small. Up to this approximation and given the list of operators above, these equations are the most general ones one can write. They reflect the fact that while DM annihilates through many channels, only a part of them $(X X \rightarrow \psi \bar{u}+$ h.c. $)$ is CP-violating (CPV) and contributes to the generation of SM and exotic baryons asymmetries. The CP-violating (CPV) interactions $\left(\gamma_{\mathrm{ann}}^{\mathrm{CP}}\right)$ are proportional to the $u$-quark number violating operators and correspond to the operators $\mathscr{O}_{1 \ldots 6}$. The CP-conserving annihilations involve the operators $\mathscr{O}_{7,8}$ and $\mathscr{O}_{13 \ldots 19}$. Further, the number asymmetries undergo washout processes. The mixed washout, $\gamma_{\text {WO }}^{m}$ (operators $\mathscr{O}_{1 \ldots 6}$ ), mix DM with $u$ - $\psi$, whereas the pure washout, $\gamma_{\text {WO }}^{p}$ (operators $\mathscr{O}_{9}$ and $\mathscr{O}_{11}$ ), involves only $u$ and $\psi$.

In equations 3.3 and 3.4, we introduced the decays of $\psi$ into two down-quarks, parametrized by the rate $\gamma_{D}$. This decay provides the only source of $d$-quark number violation. Let us note that the decays of $\psi$ inject high-energy down-quarks in the thermal bath. To avoid spoiling BigBang nucleosynthesis predictions (see, e.g. [21]), these decays have to be fast enough, with $\tau(\psi \rightarrow$ $\bar{d} \bar{d} n) \lesssim 1 \mathrm{~s}$. This places constraints on $\lambda_{n}$ that are rather weak.

\subsection{Generation of the baryon asymmetry via WIMP annihilations}

DM annihilates into a $\bar{u}$ quark and an exotic quark, $\psi$. We emphasize that in these models the DM, despite being a Dirac fermion, annihilates with itself rather than with its antiparticle. During these annihilations, CP is violated and an $u$-quark number asymmetry is created, in an equal and opposite amount to a $\psi$-number asymmetry. After being produced, the exotic quark decays into a SM singlet fermion, $n$, plus two antiquarks: $\psi \rightarrow \bar{d} \bar{d} n$. It is crucial that $\psi$ does not decay only into SM quarks, since that could eliminate the asymmetry. The $\mathbb{Z}_{4}$ symmetry prevents such dangerous decays. To avoid overclosing the Universe, $n$ has to be light. For the sake of simplicity we take it to be massless. Two scenarios are contemplated:

1. The singlet $n$ carries baryon number +1 . In this case the decay $\psi \rightarrow \bar{d} \bar{d} n$ is baryon-numberconserving, but $n$ is sequestered in a sterile sector, so we are left with a net baryon number in the visible sector. The overall process violates the SM baryon number.

2. The singlet $n$ does not carry baryon number. The decay of $\psi$ explicitly violates baryon number and it contributes to the baryon asymmetry.

Both cases satisfy the first Sakharov condition [22], that is baryon number violation. The other two conditions are also easily satisfied: $C P$ violation is achieved with complex couplings $\lambda_{i}$ and with the interference between tree-level and one-loop diagrams; departure from thermal equilibrium is automatically implemented, given that WIMP annihilation around freeze-out is out of equilibrium.

The physical CP asymmetry is defined as

$$
\varepsilon(z) \equiv \frac{\gamma(X X \rightarrow \bar{u} \psi)+\gamma(\bar{X} \bar{X} \rightarrow \bar{u} \psi)-\gamma\left(X X \rightarrow \bar{u}^{\dagger} \psi^{\dagger}\right)-\gamma\left(\bar{X} \bar{X} \rightarrow \bar{u}^{\dagger} \psi^{\dagger}\right)}{\gamma(X X \rightarrow \bar{u} \psi)+\gamma(\bar{X} \bar{X} \rightarrow \bar{u} \psi)+\gamma\left(X X \rightarrow \bar{u}^{\dagger} \psi^{\dagger}\right)+\gamma\left(\bar{X} \bar{X} \rightarrow \bar{u}^{\dagger} \psi^{\dagger}\right)} .
$$

Eq. (3.5) can be calculated in our model, at leading order, from the interference between tree-level and one-loop diagrams, as shown in figure 1 .

It is instructive to look at some limiting cases rather than at the general full expression. Setting the relations among the couplings and taking the low temperature limit $(z \rightarrow \infty)$, we find

$$
\varepsilon=\frac{\left|\lambda_{\mathrm{WO}}\right|^{2}}{4 \pi} \sin (2 \delta)\left(\frac{m_{\chi}}{\Lambda}\right)^{2}\left(1-x^{2}\right)^{2} \frac{2 \lambda_{p}^{4}+3 x \lambda_{p}^{2} \lambda_{t}^{2}+2 x^{2} \lambda_{t}^{4}}{2 \lambda_{p}^{4}-2 x \lambda_{p}^{2} \lambda_{t}^{2}+\lambda_{t}^{4}\left(1+x^{2}\right)},
$$



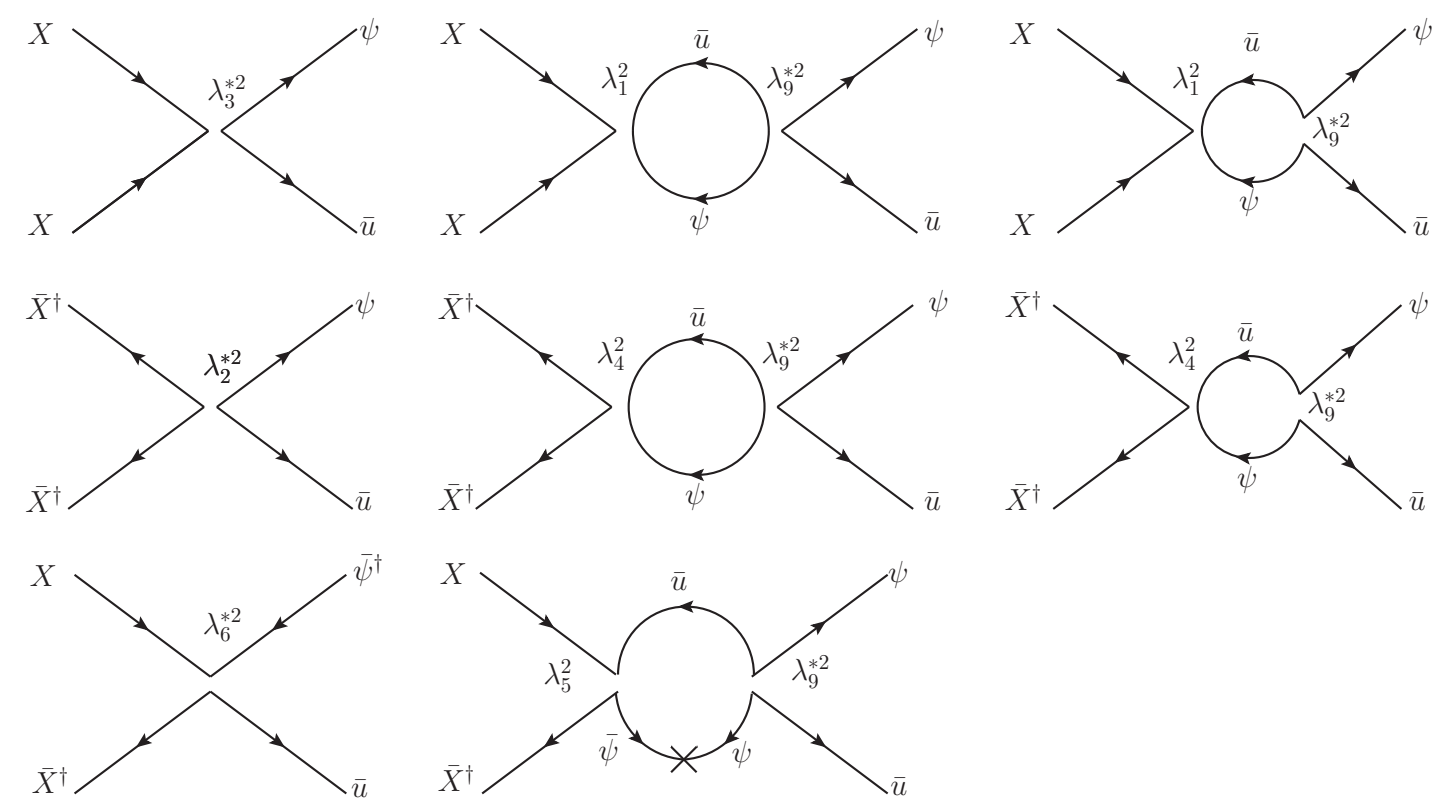

Figure 1: Diagrams for the annihilation process $X X \rightarrow \bar{u} \psi$. The diagrams in the first two lines involve $s$ channel-annihilation operators, while in the last line they involve $t$-channel-annihilation operators. The cross in the last diagram represents a mass insertion, $m_{\psi}$. The annihilation processes $X X \rightarrow \bar{u}^{\dagger} \psi^{\dagger}$ are obtained from similar diagrams, with the replacements $\lambda_{3} \rightarrow \lambda_{1}^{*}, \lambda_{2} \rightarrow \lambda_{4}^{*}, \lambda_{6} \leftrightarrow \lambda_{5}^{*}$ and $\lambda_{9} \rightarrow \lambda_{9}^{*}$.

where $x \equiv m_{\psi} /\left(2 m_{\chi}\right)$ and $\lambda_{\text {WO }}=\left|\lambda_{\text {WO }}\right| e^{i \delta} \equiv \lambda_{9}$.

To further simplify the analysis, we make the assumption that the phase is large and we set $\delta=\pi / 4$. This is not in conflict with current electric dipole moments (EDMs) measurements that would be the most constraining for these phases, given that the lowest order contribution to EDMs in these models is at three loops $[17,18]$ and so very suppressed.

A very important result, emphasized by the authors of [17], is that in order to produce a significant baryon asymmetry, washout processes must freeze out before WIMP freeze-out. To achieve this early washout freeze-out, one needs either a $\psi$ heavier than the DM, $m_{\psi}>m_{\chi}$, so that the washout is Boltzmann suppressed while DM is still annihilating, or a small couplings, such that the washout cross section is small compared to the annihilation cross section.

\section{Constraints from the LHC}

One of the new particles that we need in our models, $\psi$, is colored, which makes it a good candidate to be discovered at the LHC. Alternatively, the LHC can put severe bounds on these models. $\psi$ can be pair-produced at the hadron collider, through the process $q \bar{q} \rightarrow g \rightarrow \psi \bar{\psi}$, where a quark and an antiquark annihilate into a gluon, which then splits into $\psi$ and $\bar{\psi}$. Given that each $\psi$ decays into two (anti-)down quarks plus a singlet $n$, the signature to look for is four jets plus missing energy. Both the CMS [23] and the ATLAS [24] collaborations search for such a signature in the context of supersymmetry (SUSY), and they put bounds on the masses of gluinos and squarks from the process $p p \rightarrow \tilde{g} \tilde{g} \rightarrow 4 j+\mathbb{Z}_{T}$, where the missing energy is carried away by the lightest neutralinos. In the case of a massless neutralino, which is considered in [24], the SUSY 
process is completely analogous to the one we are interested in, with $\psi$ in place of the gluino. The production cross section differs only by a group theory factor, since $\psi$ is a color triplet, while the gluino is an octet. Once that is taken into account, we can translate the bound on the gluinos, $m_{\tilde{g}} \gtrsim 1200 \mathrm{GeV}$, into

$$
m_{\psi} \gtrsim 1000 \mathrm{GeV} \text {. }
$$

In these models DM has to annihilate into a quark, whose mass can be neglected, and an exotic antiquark, $\psi$, in order to generate a baryon asymmetry. Therefore we have the kinematical bound

$$
m_{\psi}<2 m_{\chi}
$$

Thus, the DM has to be heavier than $\sim 500 \mathrm{GeV}$.

\section{Constraints from cosmology}

In this section we assess the impact of the DM relic density and BAU constraints on our parameter space. To that end, we make use of Boltzmann equations, detailed in section 3, for the evolution of the DM density and the baryon asymmetry.

In the numerical evaluations, we fix $\Lambda=10 \mathrm{TeV}$, and we scan over $m_{\chi}$ and $m_{\psi}$, with $m_{\chi} \leq 1$ $\mathrm{TeV}$, and $800 \mathrm{GeV} \leq m_{\psi} \leq 2 m_{\chi}$. The lower and upper bounds on $m_{\psi}$ are dictated by the LHC and the kinematics of DM annihilations, respectively.

\subsection{Dark matter relic abundance}

Figure 2 shows contour levels for the coupling $\lambda_{p}$ (upper left pane), $\lambda_{t}$ (upper right pane) and $\lambda_{s}$ (lower pane) needed for generating the DM relic density abundance measured by the Planck satellite, in the $\left[m_{\psi} / m_{\chi}, m_{\chi}\right]$ plane. The white lower left region in each plot corresponds to a $\psi$ mass already ruled out by the LHC $\left(m_{\psi}<800 \mathrm{GeV}\right)$. In general, the couplings $\lambda_{p, s, t}$ have to be larger than 10. Let us remember that the scalar $s$-channel is velocity suppressed compared to the pseudoscalar one. Thus a larger $\lambda_{s}$, compared to $\lambda_{p}$ and $\lambda_{t}$, is needed to get the right relic density. We see that for a fixed $m_{\psi} / m_{\chi}$ ratio, smaller couplings are needed for larger $m_{\chi}$. Indeed, with the increase of $m_{\chi}$ the DM relic abundance gets reduced due to the thermal average (for a fixed DM annihilation cross section), and to the increase of the DM cross section itself, hence smaller couplings are required. Oppositely, for a fixed DM mass the annihilation cross section decreases for larger $\psi$ masses, therefore larger couplings are needed in order to compensate for the reduction of the available phase space.

\subsection{Baryon asymmetry}

Now we want to impose the constraint from the BAU, on top of the DM relic abundance already considered in the previous subsection. The final baryon asymmetry, that has to match the measured value, results from competing processes: $\mathrm{CP}$-violating DM annihilations generate an asymmetry, while washout processes tend to deplete it.

In figure 3 we depict contour levels for the modulus of the coupling $\lambda_{\text {wO }}$ needed for generating the measured value for the BAU, in the plane $\left[m_{\psi} / m_{\chi}, m_{\chi}\right]$, again for the limiting cases considered before. Although not shown in the plots, the values for $\lambda_{p}, \lambda_{s}$ and $\lambda_{t}$ are fixed to reproduce the 

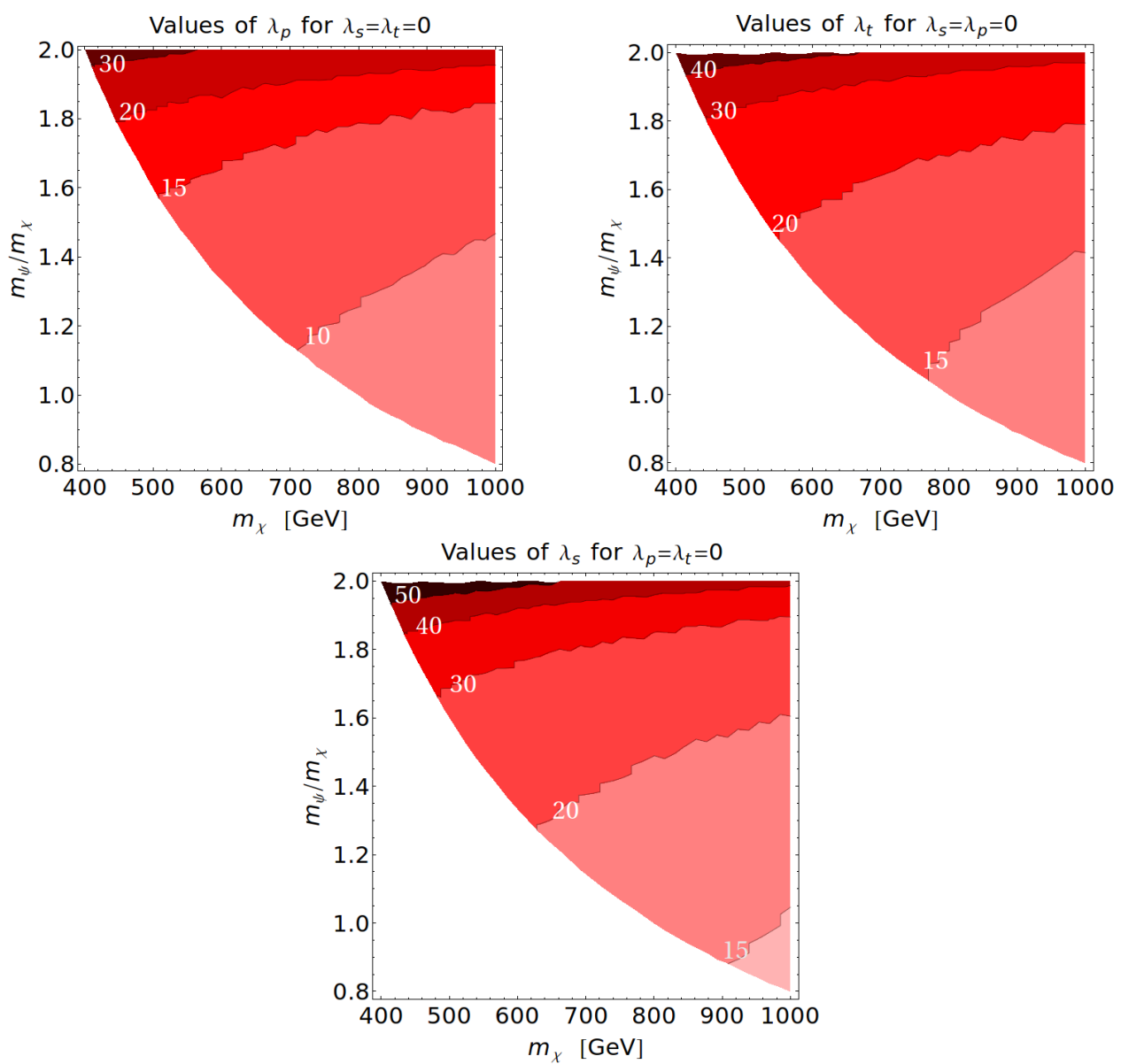

Figure 2: Contour level for the couplings $\lambda_{p}$ (upper left pane), $\lambda_{t}$ (upper right pane) and $\lambda_{s}$ (lower pane) needed for generating the DM relic density in the $\left[m_{\psi} / m_{\chi}, m_{\chi}\right]$ plane. The white lower left part corresponds to $m_{\psi} \leq 800 \mathrm{GeV}$, which is excluded by the LHC.

correct DM relic density. It is worth noticing that, except for the white region already ruled out by the LHC, the rest of the parameter space could give rise to the correct DM relic density and the baryon asymmetry, for reasonable values for the couplings. The behavior of $\left|\lambda_{\mathrm{WO}}\right|$ can be understood as follows. Recall that $\varepsilon \propto\left|\lambda_{\mathrm{WO}}\right|^{2} m_{\chi}^{2}$, while the mixed washout, which dominates over the pure one, is proportional to $\lambda_{p, s, t}^{4}$. For a fixed value of $m_{\chi}$, increasing $m_{\psi}$, both $\varepsilon$ and the washout decrease. But while the washout rates decrease quickly, $\varepsilon$ goes down slowly for $m_{\psi} / m_{\chi} \lesssim 1.8$, so $\left|\lambda_{\text {WO }}\right|$ has to decrease in this direction in order to not overproduce the asymmetry. For $m_{\psi} / m_{\chi} \gtrsim 1.8$ the washout processes are not important anymore and $\varepsilon$ would become too small, thus $\left|\lambda_{\mathrm{WO}}\right|$ has to invert the trend and start increasing. For a fixed $m_{\psi} / m_{\chi}$ ratio smaller values of $\left|\lambda_{\mathrm{wO}}\right|$ are needed when $m_{\chi}$ increases.

Figure 4 shows contour levels for $\varepsilon$ (in the low temperature limit) generated when imposing both the DM relic density and BAU constraints. The parameter $\varepsilon$ roughly follows the same behavior as if we were keeping $\left|\lambda_{\mathrm{WO}}\right|$ constant: it decreases with the increase of $m_{\psi} / m_{\chi}$ (see equation (3.6)). 

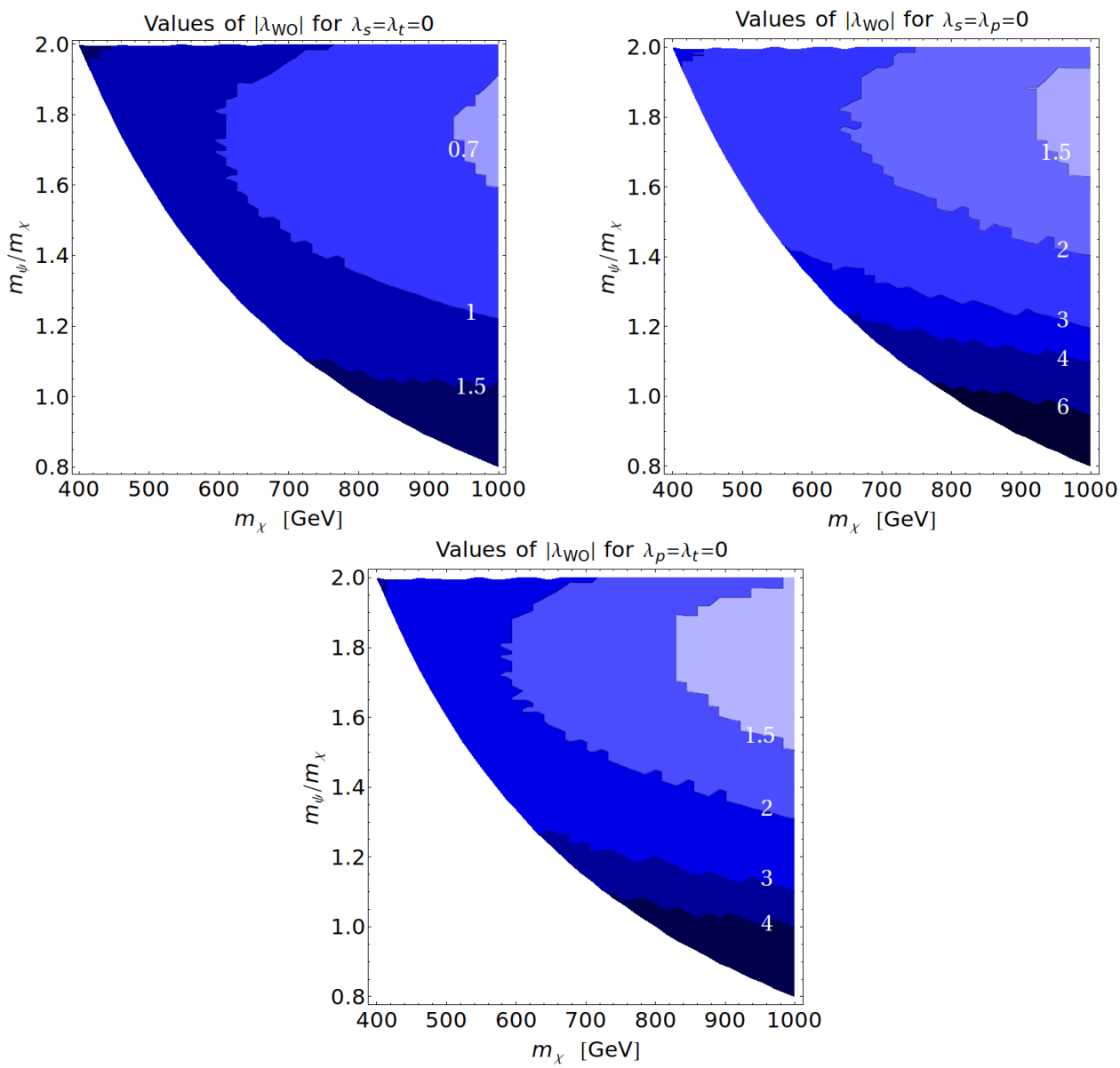

Figure 3: Contour levels for the modulus of the coupling $\lambda_{\text {Wo }}$ needed for generating the measured BAU, in the $\left[m_{\psi} / m_{\chi}, m_{\chi}\right]$ plane. We display the pseudoscalar (upper left pane), $t$-channel (upper right pane) and scalar (lower pane) cases.

\section{Constraints from dark matter direct detection}

We have two operators in our effective Lagrangian that contribute to the direct detection of DM at tree level:

$$
\frac{1}{\Lambda^{2}}\left[\lambda_{7}^{2}(X \bar{u})\left(X^{\dagger} \bar{u}^{\dagger}\right)+\lambda_{8}^{2}(\bar{X} \bar{u})\left(\bar{X}^{\dagger} \bar{u}^{\dagger}\right)\right]+\text { h.c. }
$$

In this case the spin-independent (SI) contribution is proportional to the difference of the couplings $\left(\lambda_{8}^{2}-\lambda_{7}^{2}\right)$, while the spin-dependent (SD) one to the sum $\left(\lambda_{8}^{2}+\lambda_{7}^{2}\right)$.

The operators in eq. (6.1) are also responsible for DM annihilation into a quark plus an antiquark. This annihilation channel, which does not contribute to the asymmetry, would be competing with the one into quark plus exotic anti-quark. We want the former to be suppressed with respect to the latter, in order to generate the correct BAU. Therefore, even strict bounds on the couplings $\lambda_{7}$ and $\lambda_{8}$ from direct detection, would not challenge these models. Put another way, for the WIMPy baryogenesis to work, $\lambda_{7}$ and $\lambda_{8}$ must be suppressed with respect to $\lambda_{s}, \lambda_{p}$ and $\lambda_{t}$, which would explain why no signal has been seen so far in direct detection experiments.

The impact of the XENON100 exclusion limits after 225 live days of data [25] implies that the combination of the couplings $\lambda_{7}^{2}-\lambda_{8}^{2}$ has to be smaller than $\sim 10$. If $\lambda_{7}=\lambda_{8}$, there is no 

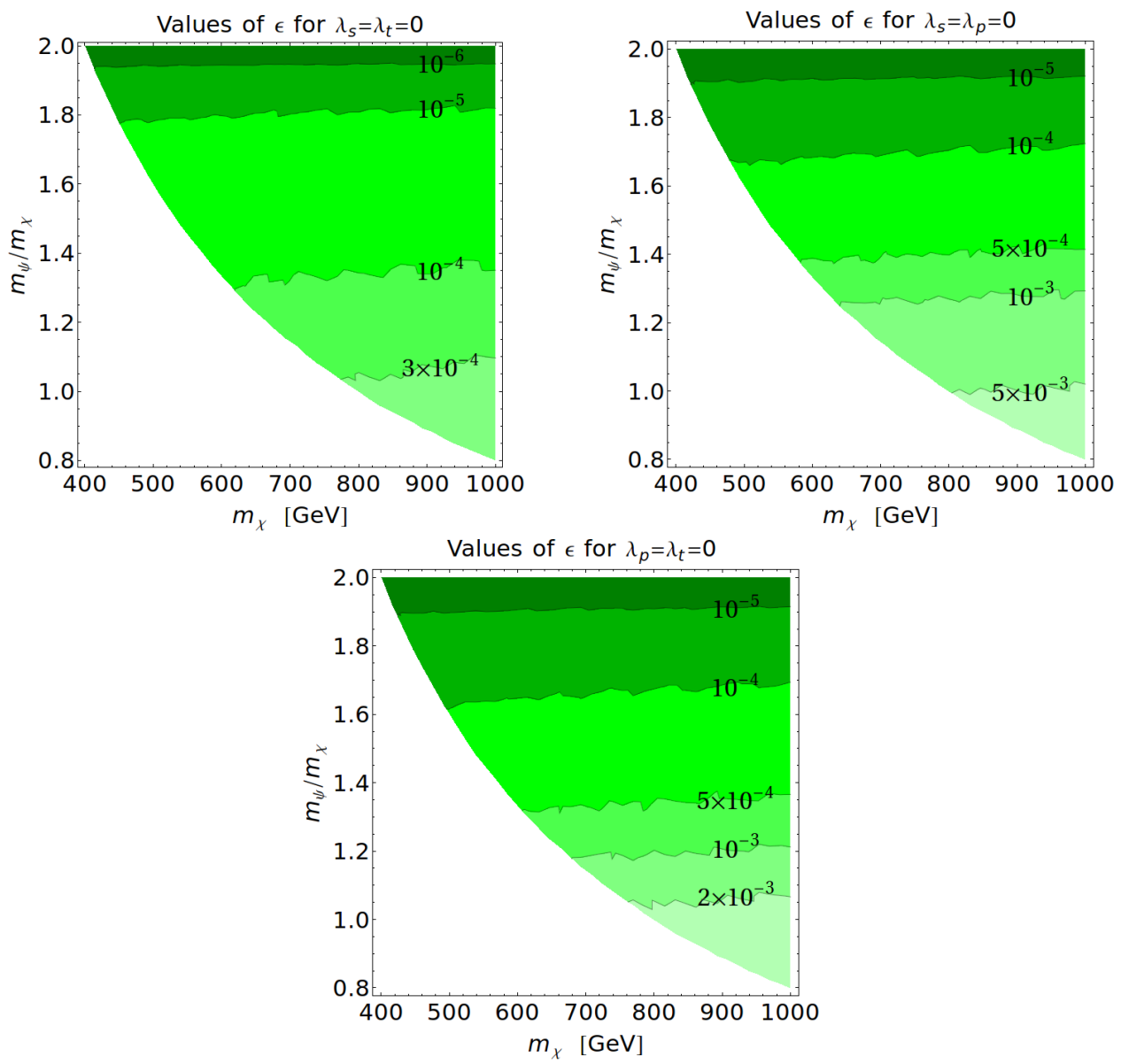

Figure 4: Contour levels for $\varepsilon$. We display the pseudoscalar (upper left pane), $t$-channel (upper right pane) and scalar (lower pane) cases.

SI contribution, but the SD one is at its maximum. The SD limits are a few orders of magnitude weaker than SI limits, and are of no interest for the present study.

\section{Summary and discussion}

In this work we have investigated whether a general class of WIMPy baryogenesis models is viable, after experimental constraints are taken into account. Our models are based on the same mechanism and on the same external particles as in Ref. [17, 18]. However, by following an EFT approach and writing down a complete list of four-fermion operators, we extend and generalize their study, considering all the possible DM annihilation channels. The models considered here require the presence of a heavy fermion, $\psi$, which is crucial to the success of the whole mechanism. Because $\psi$ is colored, the LHC represents an excellent laboratory for testing these models. Although we have not yet studied in detail possible collider signals, current LHC searches already put a lower bound of $1000 \mathrm{GeV}$ on the mass of $\psi$, which in turn directly translates into a lower bound of $500 \mathrm{GeV}$ on the DM mass. With the impressive pace at which the LHC and the ATLAS and CMS collaborations are operating, this bound can increase relative quickly, pointing to even 
higher masses, or, in a better (luckier) scenario, a heavy colored fermion could be discovered soon, which would provide a hint that these models could be realized in nature indeed.

In this work we focused mainly on the cosmological aspects and we examined in some detail the constraints from the measured DM relic density and BAU. We considered three different channels for DM annihilation into a quark and an exotic antiquark: scalar and pseudoscalar $s$-channel, and $t$-channel. We found the pseudoscalar channel to be the most promising: it has the highest annihilation cross section, the lowest washout cross section and it generates a large asymmetry $\varepsilon$. This combination results in lower values of the coupling $\lambda_{p}$, compared to $\lambda_{s}$ and $\lambda_{t}$, and in the most efficient production of the BAU. In the spirit that lower rather than higher values of the couplings are generally preferred in the EFT, our analysis, in all cases, points toward a high DM mass, between $800 \mathrm{GeV}$ and $1 \mathrm{TeV}$, and a small hierarchy between $\psi$ and $\chi, m_{\psi} \lesssim 1.4 m_{\chi}$ (see the figures in Section 5).

We also considered bounds from direct detection. These constrain only two operators that would be responsible for the annihilation of DM into a pair of quarks. Given that this channel does not contribute to the generation of the baryon asymmetry, we want it to be suppressed anyway. In this sense such bounds do not challenge these models at all. There are in principle one-loop diagrams, involving the couplings that also enter the generation of the asymmetry, that could contribute to the direct detection cross section. We showed that they are not only loop suppressed, but also velocity suppressed. Thus, this scenario is out of reach for current direct detection experiments.

Since CP violation is a crucial ingredient in these models, one has to worry that the physical phases are not too constrained. As already pointed out in [17, 18], it seems that the strongest constraints on the phases would come from EDMs measurements. For the models of $[17,18]$, the lowest order contribution to the neutron EDM only appears at three loops, and as a consequence their phases are not much constrained at all. In our EFT context, the diagrams contributing to the neutron EDM are slightly different, but it is still true that the lowest order contribution appears at three loops, so we reach the same conclusion: the $\mathrm{CP}$ violating phases are not much constrained by current experiment, so we have the freedom of taking them quite large.

\section{Acknowledgments}

We would like to thank Arindam Chatterjee, Manuel Drees, Herbi Dreiner, Marta Losada, Juan Racker, Witold Skiba and Sean Tulin for helpful discussions. NB and LU are supported by the DFG TRR33 "The Dark Universe". The work of FXJM is funded by the Fundação para a Ciência e a Tecnologia (FCT, Portugal) through the projects CERN/FP/123580/2011 and CFTP-FCT Unit 777, which are partially funded through POCTI (FEDER).

\section{References}

[1] G. Jungman, M. Kamionkowski and K. Griest, Phys. Rept. 267 (1996) 195 [hep-ph/9506380].

[2] L. Bergström, Rept. Prog. Phys. 63 (2000) 793 [hep-ph/0002126].

[3] C. Muñoz, Int. J. Mod. Phys. A 19 (2004) 3093 [hep-ph/0309346].

[4] G. Bertone, D. Hooper and J. Silk, Phys. Rept. 405 (2005) 279 [hep-ph/0404175]. 
[5] G. Bertone, Cambridge, UK: Univ. Pr. (2010) 738 p

[6] M. Drees and G. Gerbier, arXiv:1204.2373 [hep-ph].

[7] L. Bergström, Annalen Phys. 524 (2012) 479 [arXiv:1205.4882 [astro-ph.HE]].

[8] M. Tegmark et al. [SDSS Collaboration], Phys. Rev. D 74 (2006) 123507 [astro-ph/0608632].

[9] P. A. R. Ade et al. [Planck Collaboration], arXiv:1303.5076 [astro-ph.CO].

[10] S. Nussinov, Phys. Lett. B 165 (1985) 55.

[11] D. Hooper, J. March-Russell and S. M. West, Phys. Lett. B 605 (2005) 228 [hep-ph/0410114].

[12] G. R. Farrar and G. Zaharijas, Phys. Rev. Lett. 96 (2006) 041302 [hep-ph/0510079].

[13] D. E. Kaplan, M. A. Luty and K. M. Zurek, Phys. Rev. D 79 (2009) 115016 [arXiv:0901.4117 [hep-ph]].

[14] F. D'Eramo, L. Fei and J. Thaler, JCAP 1203 (2012) 010 [arXiv:1111.5615 [hep-ph]].

[15] A. B. McDonald, In *Venice 2011, Neutrino Telescopes* 1-11

[16] J. McDonald, Phys. Rev. D 84 (2011) 103514 [arXiv:1108.4653 [hep-ph]].

[17] Y. Cui, L. Randall and B. Shuve, JHEP 1204 (2012) 075 [arXiv:1112.2704 [hep-ph]].

[18] N. Bernal, F. -X. Josse-Michaux and L. Ubaldi, JCAP 1301 (2013) 034 [arXiv:1210.0094 [hep-ph]].

[19] Y. Cui and R. Sundrum, arXiv:1212.2973 [hep-ph].

[20] H. K. Dreiner, H. E. Haber and S. P. Martin, Phys. Rept. 494 (2010) 1 [arXiv:0812.1594 [hep-ph]].

[21] M. Kawasaki, K. Kohri and T. Moroi, Phys. Rev. D 71 (2005) 083502 [astro-ph/0408426].

[22] A. D. Sakharov, Pisma Zh. Eksp. Teor. Fiz. 5 (1967) 32 [JETP Lett. 5 (1967) 24] [Sov. Phys. Usp. 34 (1991) 392] [Usp. Fiz. Nauk 161 (1991) 61].

[23] [CMS Collaboration], CMS-PAS-SUS-11-016.

[24] [ATLAS Collaboration], ATLAS-CONF-2012-109.

[25] E. Aprile et al. [XENON100 Collaboration], Phys. Rev. Lett. 109 (2012) 181301 [arXiv:1207.5988 [astro-ph.CO]]. 